\title{
Electormagnetic Field Components in a Skew Uniform Magnetic Field on Kerr Newman Back Ground Geometry
}

\author{
K. N. Mishra \\ Department of Applied Mathematics, Bhilai Institute of technology, Durg-491001.India
}

\begin{abstract}
In this paper we have calculated the electromagnetic field components in a skew uniform magnetic fields superimposed on Kerr Newman back ground geometry. The rotation axis of the Kerr Newman black hole makes an angle $\gamma$ with the asymptotic magnetic field. We have compared the result with those of the corresponding Kerr black hole.
\end{abstract}

Keywords: Kerr Newman black hole, Electromagnetic field

\section{Introduction}

The Kerr Newman solutions are stationary and axisytmmetric solutions of the Einstein-Maxwell equations representing charged rotating black holes . The other stationery black hole solutions can be obtained as particular case of Kerr Newman solution. They are representative for all the black holes, because even the non stationary black holes tend asymptotically in time to Kerr Newman ones. It is well known that cosmic bodies are endowed with magnetic field and hardly any free charge ,hence astrophysically it is more relevant to look for solution of the set of Einstein - Maxwell equation with non zero magnetic field. In the case of black hole the magnetic fields are associated only with the system of currents out side the event horizon. It was originally believed that a charge black hole could not exist for long time in typical astrophysical field as selective accretion of charge could neutralize the black hole and therefore kill off magnetic field. The Maxwell equation for simple source on the space time background outside a rotating black hole, a series of article, Wald (1974), Petterson(1975) Chitre and Visheswara(1975)and King ,Lasota and Kundt(1975) indicates that the minimum energy configuration of a rotating black hole immersed into electromagnetic field of an asymptotically axis symmetric source .

The configuration of a simple axis symmetric magneto sphere about a rotating black hole attains a minimum energy configuration when the hole and the magnetosphere posses equal and opposite charge with a magnitude that is function of geometry and amount of large scale magnetic flux that threads the event horizon. This is a consequence of the dragging of inertial frame around a black hole mixing the magnetic field component with the electric field components of the Maxwell tensor. The electromagnetic field arises due to presence of charge associated with the central gravitating body. The exact solution given by Kerr- Newman family which represents the gravitational field exterior to the rotating charged black hole, featuring the three physical parameters ,the mass $M$, the charge $Q$ and the specific angulat momentum $a$.We have calculated electromagnetic field components in skew uniform magnetic field for Kerr Newman black hole.

\section{Kerr Newman Space-Time}

The Kerr- Newman space time is a stationary, axi symmetrical , and asymptotically flat solution of the EinsteinMaxwell equation. The metric In Boyer- Lindquist co ordinate takes the form

$d s^{2}=\frac{\rho^{2}}{\Delta} d r^{2}+\rho^{2} d \theta^{2}+\frac{\sin ^{2} \theta}{\rho^{2}}\left[\left(r^{2}+a^{2}\right) d \phi-a d t\right]-\frac{\Delta}{\rho^{2}}\left[a \sin ^{2} \theta d \phi-d t\right]^{2}$

With $\rho^{2}=r^{2}+a^{2} \cos ^{2} \theta$

$\Delta=r^{2}-2 M r+a^{2}+Q^{2}$

The units are chosen such that $\mathrm{c}=1$ for the speed of light and $\mathrm{G}=1$ for gravitational constant.

An important characteristic of this solution is that the source of gravity is

Surrounded by two horizons situated at a distance

$r \pm \sqrt{M^{2}-a^{2}-Q^{2}}$

The Kerr-Newman metric reduces to the Kerr metric for $Q=0$, describing the exterior of rotating non charged black hole. The Kerr Newman geometry has a horizon therefore describe a black hole if and only if $M^{2} \geq a^{2}+$ $Q^{2}$

The associated electromagnetic potential given by Bicak and Janis (1985) are as follows 


$$
\begin{gathered}
A_{t}^{B}=B_{0} \frac{a}{\rho^{2}}\left[-\rho^{2}+M r\left(1+\cos ^{2} \theta\right)\right]+B_{1} \frac{J}{\rho^{2}} \sin \theta \cos \theta(r \cos \psi-a \sin \psi), \\
A_{r}^{B}=-B_{1}(r-M) \sin \theta \cos \theta \sin \psi, \\
A_{\theta}^{B}=-B_{1}\left[\left(\operatorname{arsin}^{2} \theta+J \cos ^{2} \theta\right) \cos \psi+\left\{r^{2} \cos ^{2} \theta+\left(a^{2}-M r\right)\right\} \cos 2 \theta \sin \psi\right], \\
A_{\phi}^{B}=B_{0} \frac{\sin ^{2} \theta}{2 \rho^{2}}\left[\rho^{2}\left(r^{2}+a^{2}\right)-2 J \operatorname{ar}\left(1+\cos ^{2} \theta\right)\right] \\
\quad-B_{1} \frac{\sin \theta \cos \theta}{\rho^{2}}\left[\rho^{2} \Delta \cos \psi+M\left(r^{2}+a^{2}\right)(r \cos \psi-a \sin \psi)\right]
\end{gathered}
$$

Where $\quad \psi=\phi+\frac{a}{r_{+}-r_{-}} \ln \left(\frac{r-r_{+}}{r-r_{-}}\right) \quad$ and $\quad a=\frac{J}{M}$

The electromagnetic field which is generated when Kerr black hole is placed in originally uniform magnetic field with its direction not coinciding with the rotation axis of the hole. This solution has been given by Bicak and Janis (1985). $B_{0}^{x}, B_{0}^{y}$ and $B_{0}^{z}$ denoting the components of the oblique magnetic field in asymptotic Minkowskin coordinates as $x=r \sin \theta \cos \phi, y=r \sin \theta \sin \phi, z=r \cos \theta$. We have put $B_{0}^{y}=0$ and denote by $B_{0}^{x} \equiv B_{1}$, the field component perpendicular to the rotation axis and by $B_{0}^{z} \equiv B_{0}$, the field components aligned along the the rotation axis

We have parametrized the metric by the following three parametrs : the event horizon $r_{+}$and the innner horizon $r_{-}$and the rotation parameter $a$

The black hole mass and charge are then

$2 M=r_{+}+r_{-}, \quad Q^{2}=r_{+} r_{-}-a^{2}$ and

$$
r_{+}-r_{-}=2 \sqrt{M^{2}-a^{2}-Q^{2}}
$$

From which electromagnetic field can be calculated

\section{Asymptotically uniform magnetic fields across a rotating black hole}

The electromagnetic field $F_{i j}$ in a standard way, describe by three complex quantities

$$
\begin{aligned}
& \phi_{0}=F_{i j} l^{i} m^{j} \\
& \phi_{1}=\frac{1}{2} F_{i j}\left(l^{i} n^{j}+\bar{m}^{i} m^{j}\right) \\
& \text { and } \\
& \phi_{2}=F_{i j} \bar{m}^{i} m^{j}
\end{aligned}
$$

Where $l^{i}, n^{i}, m^{i}$ are Kinnersly (1960) complex null tetrads for Kerr Newman black hole

$$
\begin{gathered}
l^{i}=\left[\frac{r^{2}+a^{2}}{\Delta}, 1,0 \frac{a}{\Delta}\right], \\
n^{i}=\frac{1}{2 \rho \bar{\rho}}\left[\left(r^{2}+a^{2}\right),-\Delta, 0, a\right] \\
, m^{i}=\frac{1}{\sqrt{2} \bar{\rho}}\left[i \operatorname{iasin} \theta, 0,1 \frac{1}{\sin \theta}\right]
\end{gathered}
$$

With $\rho=r-i a \cos \theta$ and $\bar{\rho}=r+i a \cos \theta$

‘King and Lasota (1977) have solved Maxwell's equation and have found the general stationary solution:

$\phi_{\alpha}=\frac{2^{3 / 2}}{3} \pi i B \sum_{m=-1}^{1} \bar{Y}_{1 m}(\gamma, 0) \phi_{a}^{(1, m)}$

$$
(\alpha=0,1 \cdot 2)
$$

The stationary solution of Maxwell equation in the background of the vacuum space time in which electromagnetic field approaches asymptotically in a uniform magnetic field

On defining the electromagnetic field tensor by

$$
F_{i j}=A_{j, i}-A_{i, j}
$$


The non zero Electromagnetic Field components in Boyer -lindquist coordinates as rotation axis of the Kerr Black hole is parallel to the asymptotic magnetic field given by Wald (1974).

$$
\begin{gathered}
F_{10}=B_{0}\left[\frac{a r \sin ^{2} \theta}{\rho^{2}}-\frac{M a}{\rho^{4}}\left(r^{2}-a^{2} \cos ^{2} \theta\right)\left(1-\frac{Q}{B_{0} J}+\cos ^{2} \theta\right)\right] \\
F_{20}=B_{0} \frac{a \Delta^{1 / 2}}{\rho^{2}} \sin \theta \cos \theta \\
F_{13}=B_{0} \frac{r \Delta^{1 / 2}}{\rho^{2}} \sin \theta \\
F_{23}=B_{0} \frac{\cos \theta}{\rho^{2}}\left[\left(r^{2}+a^{2}\right)-\frac{2 m r a^{2}}{\rho^{2}}\left(1-\frac{Q}{B_{0} J}+\cos ^{2} \theta\right)\right]
\end{gathered}
$$

King and Lasota (1977) have solved Maxwell's equation and have found the general stationary solution:

$$
\phi_{\alpha}=\frac{2^{3 / 2}}{3} \pi i B \sum_{m=-1}^{1} \bar{Y}_{1 m}(\gamma, 0) \phi_{\alpha}^{(1, m)}
$$

Where $\phi_{a}^{(1, m)}$, as reported by King (1976), $\phi_{0}^{(1, m)}, \phi_{1}^{(1, m)}$ and $\phi_{2}^{(1, m)}$ for the case when magnetic field $B$ makes an angle $\gamma$ with the axis of the Kerr black hole, have been calculated exactly by Mishra and Chakraborty (1999)

$$
\begin{aligned}
\phi_{0}^{(1, m)}= & R_{1 m}(r) \xi Y_{1 m}(\theta, \phi) \\
\phi_{2}^{(1, m)}= & \frac{1}{\rho^{2}} S_{1 m}(r) \bar{\xi} Y_{1 m}(\theta, \phi), \\
& \phi_{1}^{(1, m)} \rho^{2}=-i a 2^{3 / 2} \sin \theta \xi Y_{1 m}(\theta, \phi) \int_{r_{+}}^{r} R_{1 m} d r+i a 2^{-1 / 2} \sin \theta \bar{\xi} Y_{1 m}(\theta, \phi) \int_{r_{+}}^{r} \frac{S_{1 m}}{\Delta} d r \\
& \quad-2^{-1 / 2} Y_{1 m}(\theta, \phi) \int_{r_{+}}^{r} p^{-1}\left(R_{1 m}-2 \frac{S_{1 m}}{\Delta}\right) d r
\end{aligned}
$$

We have calculate. $\phi_{0}, \phi_{2}$ exactly. The form of. The $\phi_{a}^{(1, m)}$ for the case when magnetic field $B$ makes an angle $\gamma$ with the axis of the black hole. From $\phi_{\alpha}^{(1, m)}$ we have calculated $\phi_{0}, \phi_{1}$ and $\phi_{2}$

$\phi_{0}^{(1, m)}=R_{1 m}(r) \xi Y_{1 m}(\theta, \phi)$

$\phi_{2}^{(1, m)}=\frac{1}{\rho^{2}} S_{1 m}(r) \bar{\xi} Y_{1 m}(\theta, \phi)$,

$$
\begin{aligned}
\phi_{1}^{(1, m)} \rho^{2}=-i a 2^{3 / 2} \sin \theta \xi Y_{1 m}(\theta, \phi) \int_{r_{+}}^{r} R_{1 m} d r+i a 2^{-1 / 2} \sin \theta \bar{\xi} Y_{1 m}(\theta, \phi) \int_{r_{+}}^{r} \frac{S_{1 m}}{\Delta} d r \\
-2^{-1 / 2} Y_{1 m}(\theta, \phi) \int_{r_{+}}^{r} p^{-1}\left(R_{1 m}-2 \frac{S_{1 m}}{\Delta}\right) d r
\end{aligned}
$$

For solutions regular at the horizon $\left(r_{+}\right)$one has

$$
\begin{gathered}
\text { Where } \xi=-\left(\partial_{\theta}+i \operatorname{cosec} \theta \partial_{\phi}\right), \\
\bar{\xi}=-\left(\partial_{\theta}-i \operatorname{cosec} \theta \partial_{\phi}\right) \\
Q=\frac{i a m}{\delta}
\end{gathered}
$$

$$
\begin{aligned}
& R_{1 m}=\frac{\delta^{2} 2 Q(2 Q+1) \ldots(2 Q+1)}{1.2}\left(r-r_{+}\right)^{-1+Q}\left(r-r_{-}\right)^{-1-Q} F\left[-2,1 ; 2 Q ; \frac{\left(r-r_{+}\right)}{-\delta}\right] \\
& S_{1 m}=\frac{(2 Q+2) \ldots(2 Q+1)}{2.2}\left(r-r_{+}\right)^{1+Q}\left(r-r_{-}\right)^{1-Q} F\left[0,3 ; 2+2 Q ; \frac{\left(r-r_{+}\right)}{-\delta}\right]
\end{aligned}
$$

Where $F$ is the is the standard hyper-geometric function and $Y_{1 m}(\theta, \phi)$ is the standard spherical harmonic function.

We have calculated $\phi_{0}, \phi_{2}$ and $\phi_{1}$ by using(3.17) to (3.21) for the case when $B$ makes an angle $\gamma$ with the axis of the Kerr Newman black hole 


$$
\begin{aligned}
& \phi_{0}=\frac{-B}{\sqrt{2} \Delta_{k}}\left[\sin \gamma\left\{\sin \psi \Delta_{k}+2(\operatorname{ar}-J) \cos \psi\right\}\right. \\
& \left.\quad+i\left\{\sin \gamma \Delta_{k} \cos \psi-2(\operatorname{ar}-J) \sin \psi+i \Delta_{k} \cos \gamma \sin \theta\right\}\right]
\end{aligned}
$$

The solution of $\phi_{1}$ is valid at small values of $\mathrm{r}\left(r \sim r_{+}\right)$. We take $\phi_{1}^{(l, m)}$ as given by $\phi_{1}=2^{-1 / 2} l r^{l-1} Y_{l m}(\theta, \phi)$

For our case $l=1$ we obtain

$$
\begin{aligned}
\phi_{1}=\frac{B}{2}[\cos \gamma & \left\{\frac{a}{\rho^{2}}\left(r \sin ^{2} \theta-\frac{M \Sigma_{k}\left(2-\sin ^{2} \theta\right)}{\rho^{2}}\right)+i \cos \theta\left(\frac{\left(r^{2}+a^{2}\right)^{2}-\Delta_{k} a^{2} \sin ^{2} \theta-2 a^{2}\left(2 M r-Q^{2}\right)}{\rho^{4}}\right)\right\} \\
& +\sin \gamma\left[\left(\Sigma_{k} \sin \gamma \sin \theta z\left(\operatorname{acos} \phi+2 \sin \phi \delta_{k}\right)\right)+i \omega_{k} \sin \gamma \sin \theta z(\operatorname{acos} \phi\right. \\
& \left.\left.\left.+2 \sin \phi \delta_{k}\right)\right]\right]
\end{aligned}
$$

We have started with the solution given by Bicak and Janis (1985) for the electromagnetic field which generated when an uncharged Kerr hole is placed in an originally in uniform magnetic field. The direction of which does not coincide with the hole's axis of rotation for the Maxwell field strength and the associated potential. The fields decompose into two components, $B_{0}$ and $B_{1}, B_{0}$ being the direction of the $z$ axis and $B_{1}$ along the $x$ axis.The source free Maxwell equation is a linear differential equation, so that it would admit any linear combination of particular solutions.

\section{Electromagnetic Field For Skew Symmetric Case}

The electromagnetic field components in Boyer -Lindquist coordinate for Kerr black hole given by Bicak and $\operatorname{Dvorak}(1976)$

$$
\begin{aligned}
& F_{r t}=-B_{0} \frac{J}{\rho^{4}} \Sigma_{k}\left(1+\cos ^{2} \theta\right) \\
& -B_{1} \frac{J r}{\rho^{4} \Delta} \sin \theta \cos \theta\left[\left\{r^{3}-2 M r^{2}+r a^{2}\left(1+\sin ^{2} \theta\right)+2 J a \cos ^{2} \theta\right\} \cos \psi\right. \\
& \left.-a\left\{r^{2}-4 M r+a^{2}\left(1+\sin ^{2} \theta\right)\right\} \sin \psi\right] \text {, } \\
& F_{\theta t}=-B_{0} \frac{2 J r}{\rho^{4}} \sin \theta \cos \theta\left(r^{2}-a^{2}\right) \\
& -B_{1} \frac{J}{\rho^{4}}\left(r^{2} \cos 2 \theta+a^{2} \cos ^{2} \theta\right)(a \sin \psi-r \cos \psi), \\
& F_{\phi t}=-B_{1} \frac{J}{\rho^{2}} \sin \theta \cos \theta(r \sin \psi+a \cos \psi), \\
& F_{r \theta}=-B_{1} \frac{1}{\Delta}\left[\Delta \left(B_{1}(r \sin \psi+a \cos \psi)\right.\right. \\
& \left.+a\left\{\left(M r-a^{2} \sin ^{2} \theta\right) \cos \psi-a\left(r \sin ^{2} \theta+M \cos ^{2} \theta\right) \sin \psi\right\}\right], \\
& F_{r \phi}=B_{0} r \sin ^{2} \theta-B_{1} \frac{\sin \theta \cos \theta}{\Delta}[(r \Delta-J a) \cos \psi-a(\Delta+M r) \sin \psi]-a_{\sin ^{2}} \theta F_{r t}, \\
& F_{\theta \phi}=B_{0} \Delta \operatorname{sim} \theta \cos \theta+B_{1}\left[\left(r^{2} \sin ^{2} \theta+M r \cos 2 \theta\right) \cos \psi-a\left(r \sin ^{2} \theta+M \cos ^{2} \theta\right) \sin \psi\right] \\
& -\frac{\left(r^{2}+a^{2}\right)}{a} F_{\theta t}
\end{aligned}
$$

The electromagnetic field (vector potential) due to charge is describe by the four potential $\left(A_{t}, A_{r}, A_{\theta}, A_{\phi}\right)$ 


$$
\begin{gathered}
A_{t}=A_{t}^{B}+\frac{Q}{2 M}\left[\frac{\Delta-a^{2} \sin ^{2} \theta}{\rho^{2}}\right] \\
A_{r}=A_{r}^{B} \\
A_{\theta}=A_{\theta}^{B} \\
A_{\phi}=A_{\phi}^{B}+\frac{Q}{2 M}\left[\frac{a \sin ^{2} \theta\left(r^{2}+a^{2}-\Delta\right)}{\rho^{2}}\right]
\end{gathered}
$$

The rotation axis of the Kerr Black hole is parallel to the asymptotic magnetic field Wald (1974). The non zero components of $F_{i j}$ given by Bicak and Janis (1985) are as follows

$$
\begin{aligned}
& F_{r t}=-B_{0} \frac{M a}{\rho^{4}} \Sigma_{k}\left(1-\frac{Q}{B_{0} J}+\cos ^{2} \theta\right) \\
& -B_{1} \frac{J r}{\rho^{4} \Delta} \sin \theta \cos \theta\left[\left\{r^{3}-2 m r^{2}+r a^{2}\left(1+\sin ^{2} \theta\right)+2 j a \cos ^{2} \theta\right\} \cos \psi\right. \\
& \left.-a\left\{r^{2}-4 M r+a^{2}\left(1+\sin ^{2} \theta\right)\right\} \sin \psi\right] \text {, } \\
& F_{\theta t}=-B_{0} \frac{2 J r}{\rho^{4}} \sin \theta \cos \theta\left(r^{2}-a^{2}\left(1-\frac{Q}{B_{0} J}\right)\right) \\
& -B_{1} \frac{J}{\rho^{4}}\left(r^{2} \cos 2 \theta+a^{2} \cos ^{2} \theta\right)(a \sin \psi-r \cos \psi), \\
& F_{\phi t}=-B_{1} \frac{J}{\rho^{2}} \sin \theta \cos \theta(r \sin \psi+a \cos \psi), \\
& F_{r \theta}=-B_{1} \frac{1}{\Delta}\left[\Delta\left(B_{1}(r \sin \psi+a \cos \psi)+a\left\{\left(M r-a^{2} \sin ^{2} \theta\right) \cos \psi-\left(\operatorname{arsin}^{2} \theta+J \cos ^{2} \theta\right) \sin \psi\right\}\right]\right. \\
& F_{r \phi}=B_{0} \frac{\sin ^{2} \theta}{\rho^{4}}\left[r \rho^{4}+M\left(1-\frac{Q}{B_{0} J}+\cos ^{2} \theta\right) \Sigma_{k}\right. \\
& -B_{1} \frac{J a r}{\rho^{4} \Delta} \sin ^{3} \theta \cos \theta\left[\left\{r^{3}-2 m r^{2}+r a^{2}\left(1+\sin ^{2} \theta\right)+2 J a \cos ^{2} \theta\right\} \cos \psi\right. \\
& \left.\left.-a\left\{r^{2}-4 M r+a^{2}\left(1+\sin ^{2} \theta\right)\right\} \sin \psi\right]\right] \\
& F_{\theta \phi}=B_{0} \frac{\sin \theta \cos \theta}{\rho^{4}}\left[\left(r^{2}+a^{2}\right)\left\{\left(r^{2}-a^{2}\right) \Sigma_{k}+2 a^{2} r(r-M)\left(1+\cos ^{2} \theta+2 a^{2} r \frac{Q M}{B_{0} J}\right\}-a^{2} \Delta \rho^{2} \sin ^{2} \theta\right]\right. \\
& +B_{1} r \sin ^{2} \theta[(r-m) \cos \psi-a \sin \psi] \\
& +B_{1} \frac{M \sin ^{2} \theta}{\rho^{4}}\left[\left(r^{2}+a^{2}\right) \Sigma_{k}-\rho^{2} a^{2} \cos ^{2} \theta\right](r \cos \psi-a \sin \psi)
\end{aligned}
$$

Where

$$
\begin{gathered}
z=\left(\frac{r-r_{+}}{r-r_{-}}\right) \\
a=\frac{J}{M} \\
\delta=2 \sqrt{M^{2}-a^{2}} \\
\Delta=\left(r^{2}-2 M r++a^{2}\right) \\
\Delta_{k}=\left(r^{2}-2 M r+Q^{2}+a^{2}\right) \\
\Sigma_{k}=\left(r^{2}-a^{2} \cos ^{2} \theta\right) \\
\lambda_{k}=a\left(2 r^{2}-2 M r+Q^{2}\right) \\
\delta_{k}=2 \sqrt{M^{2}-a^{2}-Q^{2}} \\
\omega_{k}=2 \operatorname{arcos}
\end{gathered}
$$

When $\gamma \neq 0$ we have calculated $F_{i j}$ using (4.11) to (4.16) as follows 


$$
\begin{aligned}
& F_{01}=F_{t r} \cos \gamma+\sin \gamma \frac{1}{2 \Delta_{k} \rho^{2}}\left[-B \cos \theta\left\{\Delta_{k} r \cos \psi-\lambda_{k} \sin \psi+a\left(\Delta_{k} \sin \psi+\frac{i J}{\delta_{k}} \cos \psi\right)\right\}\right. \\
& \left.-\frac{\Delta B}{\rho^{2}}\left\{\cos \theta-r\left(\omega_{k} \sin \psi-\Sigma_{k} \cos \theta \cos \psi\right)\right\}\right]\left\{a \sin \theta-B \operatorname{cosec} \theta \Sigma_{k} \sin \gamma \sin \theta z(a \cos \phi\right. \\
& \left.\left.+2 \sin \phi \delta_{k}\right)\right\} \\
& F_{02}=F_{t \theta} \cos \gamma+\sin \gamma\left[-B a \sin \theta \omega_{k} \sin \gamma \sin \theta z\left(\operatorname{acos} \phi+2 \sin \phi \delta_{k}\right)\right. \\
& +\frac{1}{2 \rho^{2}}\left\{\left(-B\left\{\Delta_{k} r \sin \psi+\lambda_{k} \sin \psi-\operatorname{acos}^{2} \theta\left(\Delta_{k} \cos \psi-\sin \psi \frac{i J}{\delta_{k}}\right)\right\}\right)\right. \\
& \left.\left.-\left(-\frac{B \Delta_{k}}{\rho^{2}}\left\{r \sin \psi \Sigma_{k}+\omega_{k} \cos \psi r+\operatorname{acos} \theta\left(\omega_{k} \sin \psi-\Sigma_{k} \cos \psi\right)\right\}\right)\right\}\right] \\
& F_{13}=F_{r \phi} \cos \gamma+\sin \gamma \sin \theta \llbracket-B a \Sigma_{k} \sin \gamma \sin \theta z\left(a \cos \phi+2 \sin \phi \delta_{k}\right) \\
& +\frac{\left(\mathrm{r}^{2}+\mathrm{a}^{2}\right)}{2 \Delta_{k} \rho^{2}}\left[\left(-B \cos \theta\left\{\Delta_{k} r \cos \psi-\lambda_{k} \sin \psi+a\left(\Delta_{k} \sin \psi+\frac{i J}{\delta_{k}} \cos \psi\right)\right\}\right)\right. \\
& -\left(\frac { \Delta _ { k } B } { \rho ^ { 2 } } \left\{\cos \theta\left(a \Sigma_{k} \sin \psi+\omega_{k} a \cos \theta \cos \psi\right)\right.\right. \\
& \left.\left.\left.-r\left(\omega_{k} \sin \psi-\Sigma_{k} \cos \theta \cos \psi\right)\right\}\right)\right] \rrbracket \\
& F_{23}=F_{r \phi} \cos \gamma+\sin \gamma \sin \theta \llbracket \frac{a \sin \theta}{2 \rho^{2}\left(r^{2}+a^{2}\right)}\left\{\left(-B \cos \theta\left\{\Delta_{k} r \cos \psi-\lambda_{k} \sin \psi+a\left(\Delta_{k} \sin \psi+\frac{i J}{\delta_{k}} \cos \psi\right)\right\}\right)\right. \\
& \left.-\left(\frac{\Delta_{k} B}{\rho^{2}}\left\{\cos \theta\left(a \Sigma_{k} \sin \psi+\omega_{k} a \cos \theta \cos \psi\right)-r\left(\omega_{k} \sin \psi-\Sigma_{k} \cos \theta \cos \psi\right)\right\}\right)\right\} \\
& -B \omega_{k} \sin \gamma \sin \theta z\left(a \cos \phi+2 \sin \phi \delta_{k}\right) \| \\
& F_{12}=\frac{1}{2 \Delta_{k}} \sin \gamma \llbracket\left(-B\left\{\Delta_{k} r \sin \psi+\lambda_{k} \sin \psi-\operatorname{acos}^{2} \theta\left(\Delta_{k} \cos \psi-\sin \psi \frac{i J}{\delta_{k}}\right)\right\}\right) \\
& +\left(-\frac{B \Delta_{k}}{\rho^{2}}\left\{r \sin \psi \Sigma_{k}+r \omega_{k} \cos \psi\right.\right. \\
& \left.\left.+\operatorname{acos} \theta\left(\omega_{k} \sin \psi-\Sigma_{k} \cos \psi\right)\right\}\right) \| \\
& F_{03}=\frac{1}{2 \rho^{2}} \sin \gamma \sin \theta \llbracket\left(-B \cos \theta\left\{\Delta_{k} r \cos \psi-\lambda_{k} \sin \psi+a\left(\Delta_{k} \sin \psi+\frac{i J}{\delta_{k}} \cos \psi\right)\right\}\right) \\
& +\left(\frac { \Delta _ { k } B } { \rho ^ { 2 } } \left\{\cos \theta\left(a \Sigma_{k} \sin \psi+\omega_{k} a \cos \theta \cos \psi\right)\right.\right. \\
& \left.\left.-r\left(\omega_{k} \sin \psi-\Sigma_{k} \cos \theta \cos \psi\right)\right\}\right) \|
\end{aligned}
$$

The above Kerr Newman electromagnetic $F_{i j}$ are more generalize fields which convert into the electromagnetic field describing such a field were explicitly in terms of the former solution given by Mishra and Chakraborty (1999) for $Q=0$ and $a=0$.

\section{Results And Discussion}

The Kerr- Newman metric is only the gravitational part of solution to The Einstein Maxwell equations for a full electro vacuum solution. The lines Force of electric and magnetic are measured by, electromagnetic field solutions of Maxwell equation in curved space time. we usually suppose that astrophysically relevant Electromagnetic field are weak enough so that their influence upon background geometry may be neglected. Since the Kerr metric is asymptotically flat, so electromagnetic field reduce to the original homogenous magnetic field in the asymptotic region. First such a test field was given by Wald (1974) for the special case of a perfect alignment of the asymptotically uniform magnetic field with symmetry axis. 
The general solution for arbitrary orientation of the asymptotic field was given by Bicak and Janis. (1985) we use the solution to construct the electromagnetic field around the Kerr Newman black hole in asymptotically uniform magnetic field. We have presented our investigation for the asymptotically uniform magnetic field around a Kerr Newman black hole for which rotation axis have an angle $\gamma$ with the asymptotic uniform magnetic field .The electromagnetic field arises due to Kerr Newman black hole is more generalized . The obtained electromagnetic field $F_{i j}$ fields convert into the field components were explicitly in terms of the former solution given by Mishra and Chakraborty (1999) for $Q=0$ and $a=0$. The components of electromagnetic field (4.17) to (4.22) will give more detail information for the charged rotating black hole.

\section{References}

[1]. Bicak,J and Janis,V. :Mon. Not. R. Astron. Soc. 1985 ,212, 899

[2]. Bicak.J and. Dvorak.L: Gen. Rel. Grav. 1976.7, 959

[3]. Chitre ,D.M and Vishveshwara,C.V ::1975, Phys. Rev. D12 ,153

[4]. King,A.R.:1976,Phys. Letters, 56A.339

[5]. King,A.R and Lasota.J.P.:1977Astron.Astrophy.58.175

[6]. Kinnersley,W.: J. Math. Phys. 10, 1195 (1969)

[7]. Mishra,K.N.and Chakraborty,D.K .:Astrophys. and space sci,260:1999

[8]. Petterson, J.A.:Phys. Rev. D. 12,1975

[9]. Wald, R.M.: Phys. Rev. D10, 1680 (1974) 